\title{
The use of a vest equipped with a global positioning system to assess water-contact patterns associated with schistosomiasis
}

\author{
Edmund Y.W. Seto ${ }^{1}$, Freyja Knapp ${ }^{2}$, Bo Zhong ${ }^{3}$, Changhong Yang ${ }^{4}$ \\ ${ }^{1}$ School of Public Health, University of California, Berkeley, 140 Warren Hall, Berkeley, CA 94720, USA; \\ ${ }^{2}$ Department of Landscape Architecture and Environmental Planning, College of Environmental Design, \\ 202 Wurster Hall, Berkeley, CA 94720, USA; ${ }^{3}$ Institute of Parasitic Disease, Sichuan Center for Disease \\ Control and Prevention, 6 Zhongxue Road, Chengdu, Sichuan 610041, China; ${ }^{4}$ Institute for Public Health \\ Information, Sichuan CDC, 6 Zhongxue Road, Chengdu, Sichuan 610041, China
}

\begin{abstract}
The real exposure to many of the tropical diseases is difficult to assess at the individual-level due to problems of recall, self-reported diaries, personnel requirements, and altered behaviour related to observation. We present a study in an area endemic for Schistosoma japonicum in which global positioning system (GPS) receivers were used for personal time-activity monitoring to assess water-contact associated with schistosomiasis transmission. The study subjects were equipped with a vest with an embedded GPS receiver for 8-hour periods. The resulting data were used to create hourly time-activity maps, which were subsequently used in interviews to ascertain the timing and location of the water-contacts. Based on a sample of twenty-four 8-hour person-days we found that individuals averaged $1.4 \pm 1.2$ water-contacts per day, and were surprisingly mobile, with $39 \%$ of the participants having spent time out of the village $(0.8 \pm 1.4$ hours outside of village). Such mobility suggests the need for further research into social patterns that may facilitate the spread of parasites, and contribute to sustained transmission. We present an assessment of the accuracy of cheaper commercially-available GPS units that have shown promise in such applications. We feel that a speed-filtering method is effective in managing measurement errors commonly encountered during personal activity monitoring with GPS. We conclude that personal GPS units can help reduce recall problems associated with other methods of assessing water-contact, and that they offer valuable insights into time-activity patterns that influence schistosomiasis transmission.
\end{abstract}

Keywords: global positioning system, geographical information system, exposure assessment, water-contact, Schistosoma japonicum, China.

\section{Introduction}

Assessing individual exposures to hazardous agents remains a challenge in public health research. In the context of schistosomiasis transmission, accurately quantifying where, when and how human exposure to infectious cercariae occurs is critical to

Corresponding author:

Edmund Y.W. Seto

School of Public Health

University of California, Berkeley, 140

Warren Hall, Berkeley, CA 94720, USA

Tel. +1 510642 9219; Fax +1 5106425815

E-mail: seto@berkeley.edu understanding how infection occurs and persists within populations, which sub-populations are at greatest risk, and in designing effective measures for controlling transmission. Numerous methods have been used for assessing exposure within schistosomiasis studies, the most common being self-reported exposure questionnaires and direct observation (Kloos et al., 1983, 1997; Lima e Costa et al., 1987; Chandiwana and Woolhouse, 1991; Fulford et al., 1996; da Silva et al., 1997; Ross et al., 1998a,b; Kabatereine et al., 1999; Li et al., 2000; Bethony et al., 2001; Gazzinelli et al., 2001; Scott et al., 2003; Spear et al., 2004; Seto et al., submitted). Questionnaires are problematic due to issues of recall and reliability, 
while direct observation studies are logistically difficult due to large personnel requirements for following study participants over many days. Moreover, direct observation may alter the behaviour of study participants, as would questionnaires, if participants found that certain activities required more effort on their part to report using the questionnaire forms.

Within environmental health studies, personal dosimeters are routinely used to assess exposures to poor air quality, noise and radiation. In these settings, technology provides more objective measures of exposure. From a participant's perspective, such dosimeters are often acceptable because they are typically small devices that require little effort on their part to use. Recent research has explored the use of global positioning systems (GPS) to obtain time-location data to associate with exposure data. Such studies include exposures to pesticides (Elgethun et al., 2003; Fenske, 2005), air pollutants (Riediker et al., 2003), and more general exposure (Phillips et al., 2001). There has been relatively slow adoption of these systems, perhaps due to the size, cost, complexity and battery life.

Recently, we have explored the use of both older as well as the latest versions of easy-to-wear GPS receivers to determine their feasibility in schistosomiasis exposure assessment work. We report on participants' acceptance of these systems, and based on a small sample of participant data, assess the usability of the GPS data when combined with an exposure interview. We discuss the need for error management, particularly the handling of poor GPS data when used for exposure studies. Finally, we discuss the benefits of newer systems with respect to cost and size.

\section{Materials and methods}

\section{GPS vests}

For our field study we used easy-to-wear GPS units borrowed from the children's pesticide study (Elgethun et al., 2003). These are custom-built units that cost approximately US\$2,000 per system. The units allow for continuous logging of GPS measurements (5-second intervals was used in our study), which include the time, latitude, longitude, and altitude. The time tracking potentially allows for matching of the GPS locations with other timed data recorded either from other personal exposure assessment devices like dosimeters or environmental data-loggers (temperature, humidity, etc.). Each GPS unit is nicely contained within a vest, with an antenna mounted on the shoulder, and battery pack, receiver, and data-logger in the sides of the vest (see Elgethun et al., 2003). Monitoring studies were constrained to 8 hours due to the battery-life. After measurements were recorded, the units required differential correction post-processing with separate data obtained from a nearby GPS base receiving station in order to obtain measurements with an accuracy of $1 \mathrm{~m}$, which requires additional GPS equipment or the purchase of differential correction data. The performance of these systems has been described in detail by Elgethun et al. (2003).

\section{Study area and population}

Our study was carried out in Xinmin village, production group 3, in Xichang county, Sichuan province, China, where schistosomiasis is endemic. A detailed epidemiological description of the village is available in Spear et al. (2004). With informed consent, a random sample of 12 individuals (chosen from an ID roster) agreed to wear GPS receivers on two separate days, for a total of 24 person-days of data. Each day, the GPS was given to participants in the morning, and retrieved in the evening after 8 hours of monitoring. As we retrieved the vests, we recorded each participant's reaction to wearing the vest. The participants ranged in age from 15-42 years. Only two of the 12 study subjects were women.

Time-activity maps and map-based interview

After collection of the vest, the GPS data were downloaded, and post-processed for differential 
correction with our own base station set up atop the county schistosomiasis control station $\left(102.266845^{\circ} \mathrm{E}, 27.889995^{\circ} \mathrm{N}\right.$ determined via averaging of 1 week of stationary GPS measurements), $7 \mathrm{~km}$ away in the city of Xichang. After differential correction, the GPS data were imported into geographic information systems (GIS) software on a field laptop, and overlaid on top of a $1 \mathrm{~m}$ resolution IKONOS satellite image (Space Imaging, 2003) and previously GPS-generated ditch map (Seto et al., 2001). Separate GIS maps were created for each participant. By filtering and separating the data by hour in the GIS, we produced time-activity maps that show hourly views of each person's daily activities. The maps were created overnight, so that they could be used the next day during interviews with the participants.

The hourly time-activity maps formed the basis of a new map-based interview. The morning after GPS collection, participants were interactively "walked" though the maps of their activities during the day before. The purpose of this was to aid their recall of the timing, sequence and location of their activities, and any water-contacts that had occurred. While one group participated in the interview, a different group of subjects were asked to wear the vest for the day. During the interview, we showed the subjects their trails of points indicating movements, and any clustering of points where they had stayed at particular locations to perform a particular activity. They were asked where they were going, why they were going, and how they were traveling. They were also asked what activities were carried out along the way, or if they had stopped to do anything special. In locations where we found clusters of points we were able to tell them how long they spent in certain locations based on the timing of those points. In this location, they were asked what specific activity they had performed. For each activity we asked if any water-contact had occurred, and if so, its duration. We counted only water-contact incidents that had occurred in ditch water, as opposed to contact with safe piped water.
Figure 1 shows a time-activity map created from the GPS data, showing a complete 8-hour period of activity for one participant. Each hour of activity is shown in a different colour. The approximate locations of three water-contact areas are denoted by the letters A, B and C, which were annotated onto the map during the interview.

Of the 12 participants in the study, one could not be reached for a second day of measurements. Hence, the final dataset consisted of 23 person-days.

\section{Comparison of GPS vests to newer GPS dataloggers}

Due to increasing demand for GPS-enabled consumer products, the technology has become cheaper and more energy efficient, and the devices have become smaller. While the units used in our field study were custom-made based on Trimble

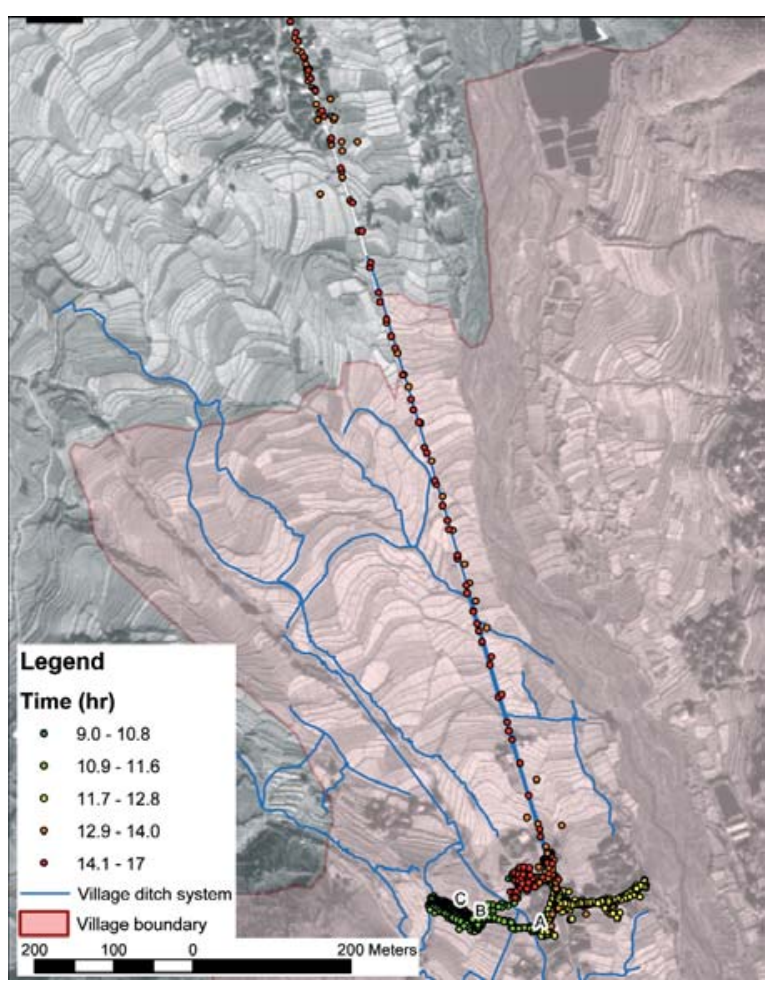

Fig. 1. Time-activity map for one participant over an 8-hour period. Labels A, B and C represent the locations of reported water contact. 
receivers, we have recently obtained commerciallyavailable GPS data-logging units based on consumergrade SiRf receivers, which can be packaged similarly into a vest for exposure assessment studies (Keskull, 2006). The new units cost about US\$ 600, and have a longer battery-life (commonly 24 hours). While the old and new units are of comparable weight $(<500 \mathrm{~g})$, the newer units are slightly smaller, which provides flexibility and alleviates bulk and balance problems associated with the earlier units. Additionally, the new units employ wide area augmentation system (WAAS) capability, a free real-time differential correction that alleviates the need for post-processing, at least in some parts of the world.

We compared the old units to the new, first by assessing measurement error for a fixed location, and second, by assessing errors along a walking path. We did not perform the indoor tests as done by Elgethun et al. (2003) as generally indoor GPS suffers from multi-path error and is not reliable. We performed all tests without the aforementioned WAAS capability as this service is not available within many of the areas where tropical diseases are prevalent.

Similar to the methods of Elgethun et al. (2003) in evaluating the older units, we placed the new units outdoors at a fixed location for more than 8 hours, with a logging interval of one measurement per second to obtain a total of 30,941 location measurements. We then, matched the data with digital orthophoto quarter quadrangle (DOQQ) imagery that clearly identified the true position of the receiver (providing a resolution with negligible $<1$ m registration error) to compute a root mean square (RMS) error. To assess performance while in motion, we walked a $670 \mathrm{~m}$ long suburban sidewalk path twice, compared the data against the true path determined from DOQQ imagery, and computed the RMS error.

\section{Results}

Statistics on activities for the 23 person-days are presented in Table 1. Certain activities such as the number and location of water-contacts can only be determined from interviews. However, other activities are easily identified simply by overlaying the data on top of IKONOS imagery. For instance, agricultural activity was identified by noting the location of activity in the terraced fields. Likewise, the time spent indoors was identified by lost signals near residence areas. Nearly all individuals spent some time indoors. Time indoors could be determined by searching for gaps in the timing of the GPS measurements (i.e. no recorded signals while individuals were indoors and out of reach from the satellite signal). The majority of participants had their lunch indoors and also spent large portions of the afternoon indoors. Time spent indoors was negatively correlated with time spent performing agricultural activities $(\mathrm{r}=-0.48, \mathrm{p}=0.02)$. These activities were clearly clustered in time and space, representing agricultural work on specific plots of land (presumably the fields they are assigned/own) within the village. On the days studied, no agricultural activity occurred outside of the village. There were a surprising number of individuals who ventured outside of the village for other reasons though (close to $40 \%$ of the person-days). Frequently these trips were north to the township square. However, two participants traveled south to a neighboring village in another county. Water-contacts were only slightly positively correlated with agricultural activity $(\mathrm{r}=0.17, \mathrm{p}=0.43)$. While the mean number of contacts per person-day was 1.4 contacts, six interviews reported no contacts at all, while one interview resulted in the maximum of four contacts.

After the first day of our study, two of the six participants complained about the bulk of the units they had been equipped with. The participants were maintaining their fields, which involved use of a hoe (Fig. 2). Because we configured the vest with the battery/receiver unit in the front pocket, the weight of the vest was not balanced, which we realized was awkward given the agricultural activities that the villagers were performing. After reconfiguring the vest to balance the weight of the system by placing the receiver and batteries in a pocket in the middle 
Table 1. Statistics on activities identified from interviews and interpretation of GPS locations ( $\mathrm{N}=23$ person-days).

\begin{tabular}{lccc}
\hline Activity & $\begin{array}{l}\text { No. persons-days } \\
\text { performing the activity }{ }^{1}\end{array}$ & Mean $\pm \mathrm{SD}^{2}$ & $\begin{array}{c}\text { Determined from } \\
\text { interview (I) or map (M) }\end{array}$ \\
\hline Water contact & $17(74 \%)$ & $1.4 \pm 1.2$ contacts & $\mathrm{I}$ \\
Agricultural activity & $18(78 \%)$ & $1.5 \pm 1.8$ hours & $\mathrm{M}$ \\
Indoor activity & $21(91 \%)$ & $2.3 \pm 2.0$ hours & $\mathrm{M}$ \\
Activity outside of village & 9 & $0.8 \pm 1.4$ hours & $\mathrm{M}$ \\
\hline
\end{tabular}

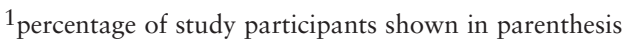

$2 \mathrm{SD}=$ standard deviation

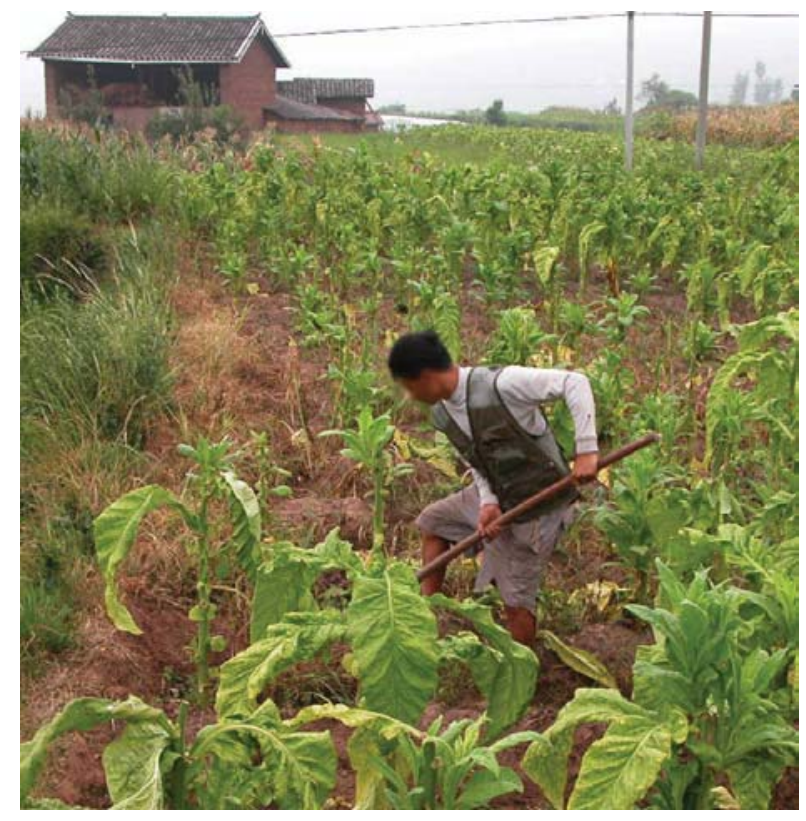

Fig. 2. Participant wearing GPS vest while performing routine agricultural activity.

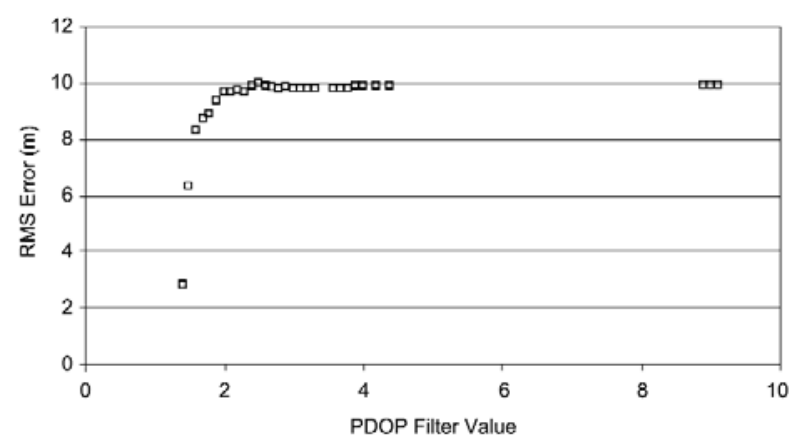

Fig. 3. Effect of PDOP filtering. RMS error computed for all points with PDOP less than or equal to the filter value. of the back of the vest, no further complaints were documented on the packaging of the system. Indeed, one participant found the vest so comfortable that he went into the city in the afternoon forgetting to return the vest which was retrieved by us the next morning.

The test of the new GPS units initially revealed that in a stationary position, the locations did not vary at all, with coordinates that remained the same for all measurements due to the SiRF III chipset's static navigation circuitry, which holds the position fixed while the receiver is not moving. Although this significantly reduces the jaggedness of GPS measurements compared to the older units, slow movement may not be recorded accurately. Turning off static navigation resulted in a RMS error of $9.9 \mathrm{~m}$. This was roughly three times worse than the older GPS-PAL units, which had RMS errors of $3.0 \mathrm{~m}$ and $3.4 \mathrm{~m}$ for two successive tests. For the walking test, RMS error was $1.0 \mathrm{~m}$, which bettered the GPS-PAL units, for which only $79 \%$ of points were within 2 $\mathrm{m}$ of the reference walking line.

To determine the effect of satellite geometry on position error, we selectively filtered measurements with varying levels of position dilution of precision (PDOP). PDOP is a common GPS indicator of the quality of the 3-dimensional measurement based on satellite positioning overhead, with high PDOP values associated with less accurate measurements. Figure 3 illustrates the effect of filtering out high PDOP measurements on RMS error. Although the data suggests that it is possible to reduce RMS error to $<6.3 \mathrm{~m}$ if we filter out measurements with PDOP higher than 1.5, in actual practice, in the suburban environment 
(nearby 2-storey buildings, similar to a village environment), only $3 \%$ of the measurements achieved these low PDOP levels. Roughly half of the data would be filtered out with a PDOP filter of 1.9-2.0, which would improve the RMS errors by approximately $0.5 \mathrm{~m}$.

Large errors were frequently found in both the field data collected with the older GPS units, as well as with the newer units. These errors may be due to temporary coverage, poor positioning of the GPS antenna, or interference. The errors are easily identified however, using the time indications associated with the measurements. By looking at successive measurements in time, it is possible to see where the measurements suddenly jump to a distant nonsensical location, and then back to where the data continue from before. Hence, we attempted a novel error reduction method based on a speed filtering procedure. The procedure is based on a comparison of the distance (or speed) traveled between successive GPS measurements. If the speed traveled is greater than a specified threshold filter value, we removed the measurement. We explored thresholds of $0.25,0.5,0.75$, and $1.0 \mathrm{~m} / \mathrm{s}$. In comparison to the PDOP filter, the results of the speed filtering method were quite effective. For the newer GPS units, the four speed filters resulted in reduction of the $9.9 \mathrm{~m}$ overall RMS error to 8.8, 9.3, 9.6, and $9.8 \mathrm{~m}$, respectively. However, considerably fewer measurements were discarded in achieving these error reductions than via PDOP filtering $(33 \%, 10 \%, 4 \%$, and $1 \%$, respectively).

\section{Discussion}

This study demonstrates the utility of GPS receivers in monitoring personal activity patterns that affect schistosomiasis risk. These activity patterns become particularly relevant to the transmission process when correlated with spatio-temporal patterns of total and infected snail density and/or cercarial concentration data (Seto et al., 2001; Spear et al., 2004). For example, in a recent study, we found that the spatial location of water-contacts is crucial to estimating exposures, and that these exposures can explain individual-level variations in infection (Seto et al., submitted).

Although we did not formally assess the recall, we did notice that participants commonly did not remember having any water-contact at all until we talked them through the recorded daily activities and reminded them that they spent time in certain locations. The activity maps demonstrate that villagers have relatively unstructured daily activities, spending typically less than two hours on average in their fields, at least during non-planting/harvesting seasons. Hence, it is understandable that they may not remember the exact day-to-day activities, when they conducted certain tasks during the day and in what order. By using the maps to help reconstruct the ordering and location of their activities, it may be easier for them to remember water-contact which surely is of less importance to them than it is to us. Hence, we believe that the map-based interviews can serve as a means to reduce recall errors.

Despite being a small dataset, we found unexpected results from the activity data. One of the major findings was the high level of mobility exhibited by the participants in this study. Although the township square was $3.5 \mathrm{~km}$ away from the village, it was not uncommon for villagers to spend time there. Presumably, the township serves as a socio-economic hub bringing together residents from nearby villages to buy and sell goods and interact socially. Such mobility patterns raise concern as to the degree to which agricultural labour is shared across villages, and if water-contact occurs not only in one's own village, but regionally. Theoretically, such exchanges can sustain transmission of schistosomiasis, and make control more difficult (Seto and Gurarie, 2006). Indeed, there is anecdotal evidence that such labour exchanges do occur. However, more research is warranted to evaluate the risk of such behaviours.

Some potential problems were encountered during our study. For instance, if the need arises to 
locate the water-contact activity precisely in the future, polygons may be drawn during the interview (Fig. 1). Another weakness was highlighted when one of the participants felt cold one day and put a jacket over the vest blocking the signal to the GPS antenna. Such problems may be solved through better instruction of the participants. Other errors, such as atmospheric interference and satellite geometry issues, may be managed via the methods presented, including PDOP and/or speed filtering. With the speed filtering method, we showed that that the large distances between successive measurements are obvious indicators of a poor measurement that can be filtered, and that this performs better by discarding less data than a PDOP filter. It also suggests that there is information to be gained by using the most frequent sampling interval possible. Other problems encountered in our study include the unequal male/female participation. Because men and women may differ with regard to their exposure, future studies with relatively small numbers should preferably prescreen potential participants and stratify by criteria such as sex, age, occupation, etc. Indeed, even the small number of females of the present study hinted at some differences between men and women, with men, on average, 1.7 times more likely to venture outside the village, spending approximately one less hour per day indoors, and having almost one water-contact less per day (however, $\mathrm{p}>0.5$ for all). Another potential uncertainty is related to whether participants changed behaviour due to the wearing of the vests. We noted in our study that initial participants complained that the units were unbalanced, and thus may have avoided certain activities in the field. While we did not attempt a comparison of behaviour between individuals wearing vests versus not wearing them, such biases may be difficult to quantify. We would then have to rely on either direct observation or diaries, both of which also have the potential to alter behaviour, perhaps to an even greater degree. We do believe, however, that as the devices become smaller and lighter, participants may prefer GPS methods as they are less immediately obstructive to their daily activities.

Although GPS vests seem to be useful in estimating behaviours that influence schistosomiasis transmission, and the cost associated with the units themselves has decreased dramatically with the commercial availability of consumer-grade data-logging GPS units, the question of whether such devices are feasible for large-scale studies depends on whether GPSbased assessments can be practically administered. Technological developments have made newer units smaller and more energy-efficient, providing more flexibility. Even though new units allow for larger numbers to be used in a study, there is a limitation in that the data from the devices must be processed into time-activity maps, which then must be interpreted through interviews or perhaps simpler and more structured questionnaires. On the other hand, the production of time-activity maps could be automated such that the creation of the maps takes less than 10 minutes per study subject. In fact, during our field study, even without automated methods for generating GIS maps, we simply divided the map-making task between two persons with GIS skills to halve the time of processing. Likewise, while the interview process cannot be automated, training a crew of fieldworkers to administer the GPS study can make the study of large numbers, for instance, $20 \%$ of a village (roughly 50 persons) feasible within a research study.

In conclusion, we feel that personal GPS units can help reduce recall problems associated with other methods of assessing water-contact, and that they offer valuable insights into time-activity patterns that influence schistosomiasis transmission.

\section{Acknowledgements}

We thank Professor Robert Spear from UC Berkeley and Michael Yost, and Wenjie Zhu from the University of Washington. EYWS was supported in part by the National Institutes of Health (NIAID RO1 AI50612). We also thank the anonymous reviewers for their comments and suggestions for improving the manuscript. 


\section{References}

Bethony J, Williams JT, Kloos H, Blangero J, Alves-Fraga L, Buck G, Michalek A, Williams-Blangero S, Lo Verde PT, Correa-Oliveira R, Gazzinelli A, 2001. Exposure to Schistosoma mansoni infection in a rural area in Brazil. II: household risk factors. Trop Med Int Health 6, 136-145.

Chandiwana SK, Woolhouse ME, 1991. Heterogeneities in water contact patterns and the epidemiology of Schistosoma haematobium. Parasitology 103, 363-370.

da Silva AA, Cutrim RN, de Britto e Alves MTeAMT, Coimbra LC, Tonial SR, Borges DP, 1997. Water-contact patterns and risk factors for Schistosoma mansoni infection in a rural village of northeast Brazil. Rev Inst Med Trop Sao Paulo 39, 91-96.

Elgethun K, Fenske RA, Yost MG, Palcisko GJ, 2003. Timelocation analysis for exposure assessment studies of children using a novel global positioning system instrument. Environ Health Perspect 111, 115-122.

Fenske RA, 2005. State-of-the-art measurement of agricultural pesticide exposures. Scand J Work Environ Health 31, 67-73; discussion 63-65.

Fulford AJ, Ouma JH, Kariuki HC, Thiongo FW, Klumpp R, Kloos H, Sturrock RF, Butterworth AE, 1996. Water contact observations in Kenyan communities endemic for schistosomiasis: methodology and patterns of behaviour. Parasitology 113, 223-241.

Gazzinelli A, Bethony J, Fraga LA, LoVerde PT, CorreaOliveira R, Kloos H, 2001. Exposure to Schistosoma mansoni infection in a rural area of Brazil. I: water contact. Trop Med Int Health 6, 126-135.

Kabatereine NB, Vennervald BJ, Ouma JH, Kemijumbi J, Butterworth AE, Dunne DW, Fulford AJ, 1999. Adult resistance to schistosomiasis mansoni: age-dependence of reinfection remains constant in communities with diverse exposure patterns. Parasitology 118, 101-105.

Keskull RI, 2006, GPS Data Logger. Available: http://homepages.tig.com.au/ robk/datalogger.html (accessed: $15 \mathrm{Fe}-$ bruary 2007).

Kloos H, Fulford AJ, Butterworth AE, Sturrock RF, Ouma JH, Kariuki HC, Thiongo FW, Dalton PR, Klumpp RK, 1997. Spatial patterns of human water contact and Schistosoma mansoni transmission and infection in four rural areas in Machakos district, Kenya. Soc Sci Med 44, 949-968.
Kloos H, Higashi GI, Cattani JA, Schlinski VD, Mansour NS, Murrell KD, 1983. Water contact behavior and schistosomiasis in an upper Egyptian village. Soc Sci Med 17, 545-562. Li YS, Sleigh AC, Williams GM, Ross AG, Forsyth SJ, Tanner M, McManus DP, 2000, Measuring exposure to Schistosoma japonicum in China. III. Activity diaries, snail and human infection, transmission ecology and options for control. Acta Trop 75, 279-289.

Lima e Costa MF, Magalhaes MH, Rocha RS, Antunes CM, Katz N, 1987. Water-contact patterns and socioeconomic variables in the epidemiology of schistosomiasis mansoni in an endemic area in Brazil. Bull World Health Organ 65, 57-66.

Phillips ML, Hall TA, Esmen NA, Lynch R, Johnson DL, 2001. Use of global positioning system technology to track subject's location during environmental exposure sampling. J Expo Anal Environ Epidemiol 11, 207-215.

Riediker M, Williams R, Devlin R, Griggs T, Bromberg P, 2003. Exposure to particulate matter, volatile organic compounds, and other air pollutants inside patrol cars. Environ Sci Technol 37, 2084-2093.

Ross AG, Sleigh AC, Li YS, Williams GM, Waine GJ, Forsyth SJ, Yi L, Hartel GF, McManus DP, 1998a. Measuring exposure to S. japonicum in China. II. Activity diaries, pathways to infection and immunological correlates. Acta Trop 71, 229-236.

Ross AG, Li YS, Sleigh AC, Williams GM, Hartel GF, Forsyth SJ, Yi L, McManus DP, 1998b. Measuring exposure to S. japonicum in China. I. Activity diaries to assess water contact and comparison to other measures. Acta Trop 71, 213-228.

Scott JT, Diakhate M, Vereecken K, Fall A, Diop M, Ly A, De Clercq D, de Vlas SJ, Berkvens D, Kestens L, Gryseels B, 2003. Human water contacts patterns in Schistosoma mansoni epidemic foci in northern Senegal change according to age, sex and place of residence, but are not related to intensity of infection. Trop Med Int Health 8, 100-108.

Seto EYW, Gurarie D, 2006. Modeling schistosomiasis transmission in a distributed environment: implications for sustainable control. American Society for Tropical Medicine and Hygiene 55th Annual Conference, Atlanta, GA, USA.

Seto EYW, Lee EY, Liang S, Zhong B, 2007. Individual and village-level study of water contact exposure patterns and Schistosoma japonicum reinfection in mountainous rural 
China. Trop Med Int Health, submitted.

Seto EYW, Liang S, Qiu D, Gu X, Spear RC, 2001. A protocol for geographically randomized snail surveys in schistosomiasis fieldwork using the global positioning system. Am J Trop Med Hyg 64, 98-99.

Space Imaging, 2003. IKONOS imagery products and prod- uct guide.

Spear RC, Seto E, Liang S, Birkner M, Hubbard A, Qiu D, Yang C, Zhong B, Xu F, Gu X, Davis GM, 2004. Factors influencing the transmission of Schistosoma japonicum in the mountains of Sichuan province of China. Am J Trop Med Hyg 70, 48-56. 